\title{
Mapping Local Photocurrents in Polymer/Fullerene Solar Cells with Photoconductive Atomic Force Microscopy
}

David C. Coffey, Obadiah G. Reid, Deanna B. Rodovsky, Glenn P. Bartholomew, and David S. Ginger* Department of Chemistry, University of Washington, Seattle 98195-1700

ginger@chem.washington.edu

\section{Supporting Information}

\section{Experimental Methods:}

Devices were fabricated on ITO coated glass substrates (TFD Inc.). To define contacts, the device area of the ITO was covered in tape, and the remainder etched away in a solution of $50 \mathrm{mg}$ Zinc dissolved in $40 \mathrm{~mL}$ of $1.2 \mathrm{M} \mathrm{HCl}$. The substrates were rinsed and then cleaned by sonication in isoproponal for 20 minutes, by sonication in acetone for 20 minutes, and by air-plasma cleaning for 5 minutes. A $40 \mathrm{~nm}$ thick PEDOT:PSS (Baytron® P VP Al 4083, filtered with $0.45 \mu \mathrm{m}$ PVDF filter) layer was first deposited, followed by an $\sim 80 \mathrm{~nm}$ active layer of MDMO-PPV:PCBM, and then a thermally evaporated, $40 \mathrm{~nm}$-thick aluminum top-contact. The PEDOT-PSS was spin-coated at 4000 RPM and annealed under nitrogen at a temperature of $110 \mathrm{C}$ for 30 minutes. PCBM was purchased from American Dye Source (Quebec, Canada). Except for the films studied in figure 3, MDMO-PPV (Mn= 537,000 and Mw/Mn = 1.03) was synthesized in our laboratory using a Gilch polymerization route via dehydrohalogenation of appropriate precursors as outlined in the literature ${ }^{1}$. The MDMO-PPV used for the device studied in figure 3 was purchased from Aldrich. The in-house MDMO-PPV devices exhibited an efficiency approximately 10 times higher than the devices fabricated with Aldrich MDMO-PPV. Blend solutions 
of MDMO-PPV and PCBM were prepared at a polymer concentration of $0.3 \% \mathrm{wt} . / \mathrm{vol}$. in chlorobenzene or xylenes, and spin coated at an elevated temperature of $40^{\circ} \mathrm{C}$. In order to maintain equal thicknesses, chlorobenzene solutions were spin-coated at $2500 \mathrm{rpm}$, while xylenes solutions were spin coated at 3000 rpm. Before deposition of the aluminum electrodes, all samples were kept under a vacuum of $10^{-6} \mathrm{mB}$ for 12 hours to remove any residual solvents. Aluminum was evaporated through a shadow mask at a rate of $0.2 \mathrm{~nm} / \mathrm{sec}$, defining devices $1.5 \mathrm{~mm}^{2}$ in area. Bulk device measurements were made in a custombuilt vacuum chamber reaching $5 \mathrm{mB}$ under an average illumination intensity of $10 \mathrm{Wm}^{-2}$. Photocurrent action spectra and current-voltage characteristics were read using a digital source-measure unit (Keithley model 2400). EQEs were calculated by taking the ratio of a device photocurrent to that of a corrected Siphotodiode with known spectral response.

Photoconductive atomic force microscopy (pcAFM) was performed in the areas between the aluminum electrodes after device measurements. Samples were imaged with an atomic force microscope (Asylum Research, MFP-3D, Santa Barbara, CA) within a nitrogen flow cell positioned above an inverted optical microscope. All reported voltages are the bias applied to the ITO substrate. Platinumcoated, contact-mode AFM tips were used, with manufacturer specified tip diameters less than $25 \mathrm{~nm}$ (Budget Sensors, BS-ElectriCont). The height and current was measured simultaneously in contact mode. Currents were measured by the AFM's internal current preamplifier (Asylum Research ORCA model 59) positioned just above the AFM tip. To measure photocurrents, a $5 \mathrm{~mW}, 532 \mathrm{~nm}$ laser or a 20 $\mathrm{mW}, 405 \mathrm{~nm}$ laser (Crystal Laser, Reno NV) were focused onto the substrate and aligned to the tip as viewed with the optical microscope. More precise alignment and focusing was achieved by maximizing the photocurrent. Illumination intensities between $\sim 10$ and $\sim 10^{9} \mathrm{Wm}^{-2}$ could be selected by a series of neutral density filters, including a neutral density filter continuously variable between optical density 0 and 4. The position and thus transmission through this filter was precisely controlled by a stepper motor. The relative light intensity was continuously monitored by splitting a small fraction of the laser light to a Si-photodiode. Absolute intensities were calculated by measuring the power exiting the 


\section{Lack of Dependence on Illumination Wavelength}
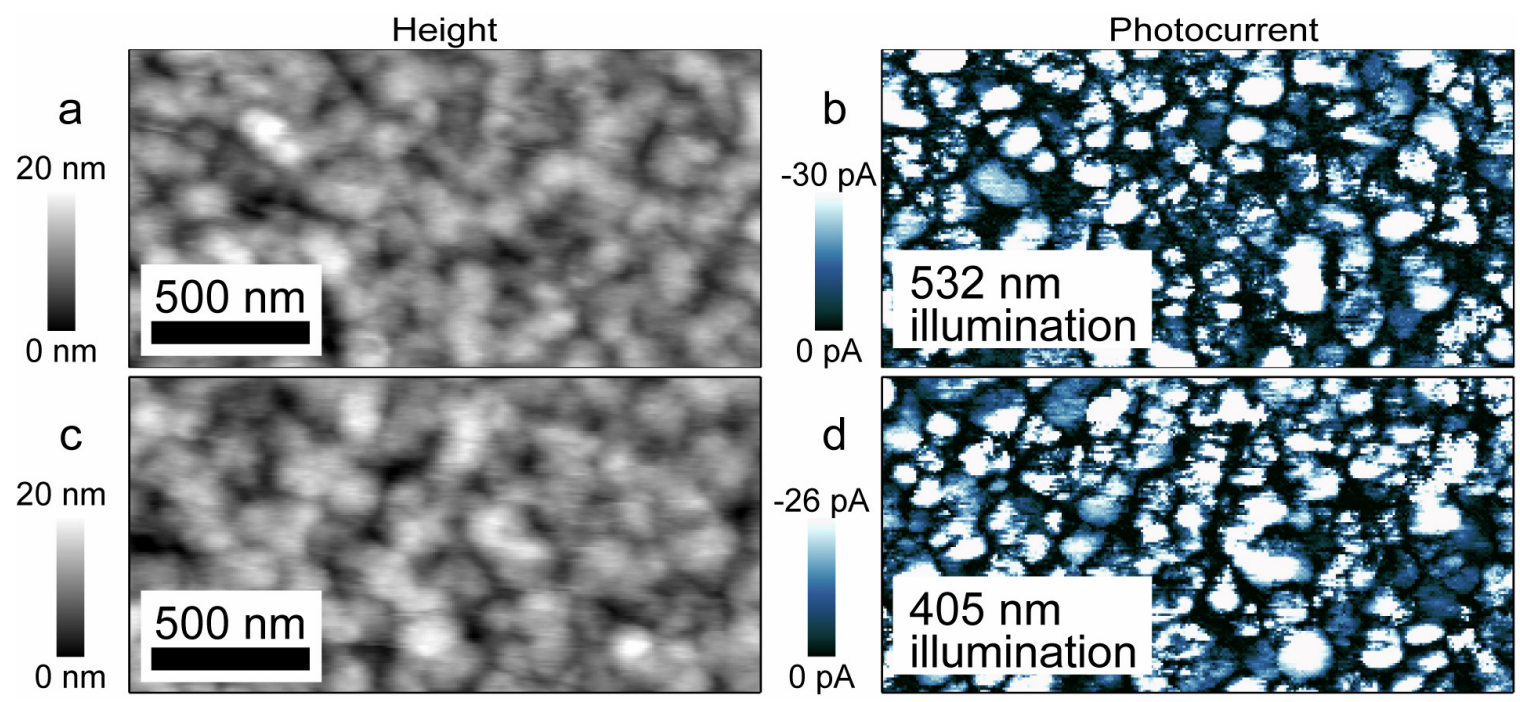

Figure S1. AFM height (a and c) images of a MDMO-PPV:PCBM (20:80) film spin coated from xylenes. Photocurrent images acquired with zero bias, with a $532 \mathrm{~nm}$ laser, b, and with a $405 \mathrm{~nm}$ laser, $\mathrm{d}$, show similar contrast, suggesting the photocurrent variations are less related to spatial absorbance variations.

\section{Comparison of device EQEs and pcAFM photocurrents}

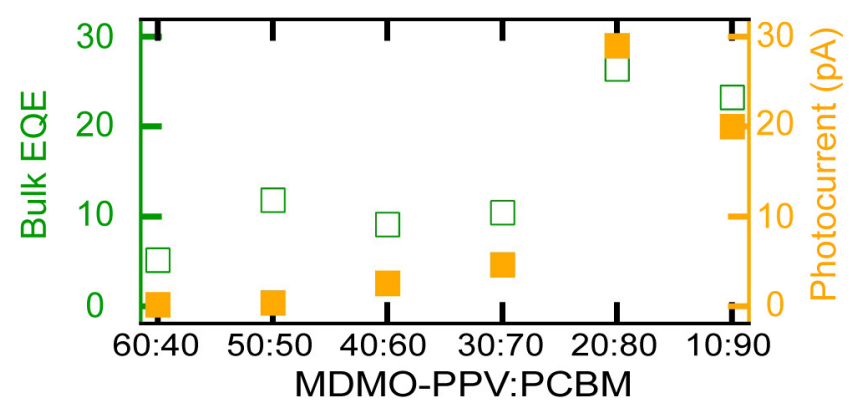

Figure S2. a) The EQEs measured for bulk, MDMO-PPV:PCBM devices spin coated from chlorobenzene with varying blend ratios (open squares) are overlaid with the short-circuit photocurrent 
averaged over $2 \times 2 \mu \mathrm{m}^{2}$ images (filled squares). Though pcAFM tends to slightly over-predict performance for devices enriched in PCBM and underpredict performance for devices rich in MDMOPPV, the overall correlation between pcAFM signal and device photocurrent remains good.

Supporting Information References:

(1) Becker, H.; Spreitzer, H.; Ibrom, K.; Kreuder, W., Macromolecules, 1999, 32, (15), 4925. 